\section{BY ERIC HAND}

$\mathrm{W}$ hen astronomer Alan Dressler was invited to see what might be the future of NASA's astrophysics programme, he had to leave his mobile phone behind, lest he be tempted to grab a quick snapshot. As he and a dozen others passed through the ITT Exelis facility in Rochester, New York, a guide held up a flashing red light, to warn working Exelis engineers to seal their lips in front of people without security clearance.

Their destination was the cavernous clean area of Building 1230, where two 2.4-metre telescopes, each as big as the Hubble Space Telescope and never flown, rested on low pedestals. "It seemed almost too good be true," says Dressler, an astronomer at the Carnegie Observatories in Pasadena, California. "Things like this just don't drop on your doorstep."

The unexpected gift comes from the US National Reconnaissance Office (NRO), a secretive surveillance agency that built the telescopes to peer down on Earth. In June, NASA revealed that the NRO had bequeathed the scopes to the space agency because they were no longer needed. Now NASA has to figure out what it will do with them - and whether it can afford the cost of kitting them out with instruments and sending them into orbit.

This month, NASA plans to announce a science-definition team that will embark on that assessment. The team will report by April 2013 to NASA administrator Charles Bolden on the pros, cons and costs of adapting one of the telescopes for a mission to investigate dark energy, the phenomenon thought to be accelerating the expansion of the Universe. But astronomers are already encouraged. As the veil of secrecy surrounding the telescopes lifts, astronomers are beginning to size up the devices' capabilities. And so far, they are liking what they see - so much so that they are now talking about tacking on an instrument that would detect extrasolar planets directly. "I think the enthusiasm has only increased as time has gone on," says Dressler.

The most likely first use for an NRO telescope is as an alternative to the proposed Wide-Field

\section{$\rightarrow$ NATURE.COM}

For more on space telescopes see: go.nature.com/vy7sy8

\title{
ANATOMY OF A GIFT
}

The National Reconnaissance Office (NRO), a US spy agency, has given NASA two telescopes, each with a primary mirror as big as that in the Hubble Space Telescope but with a wider field of view.

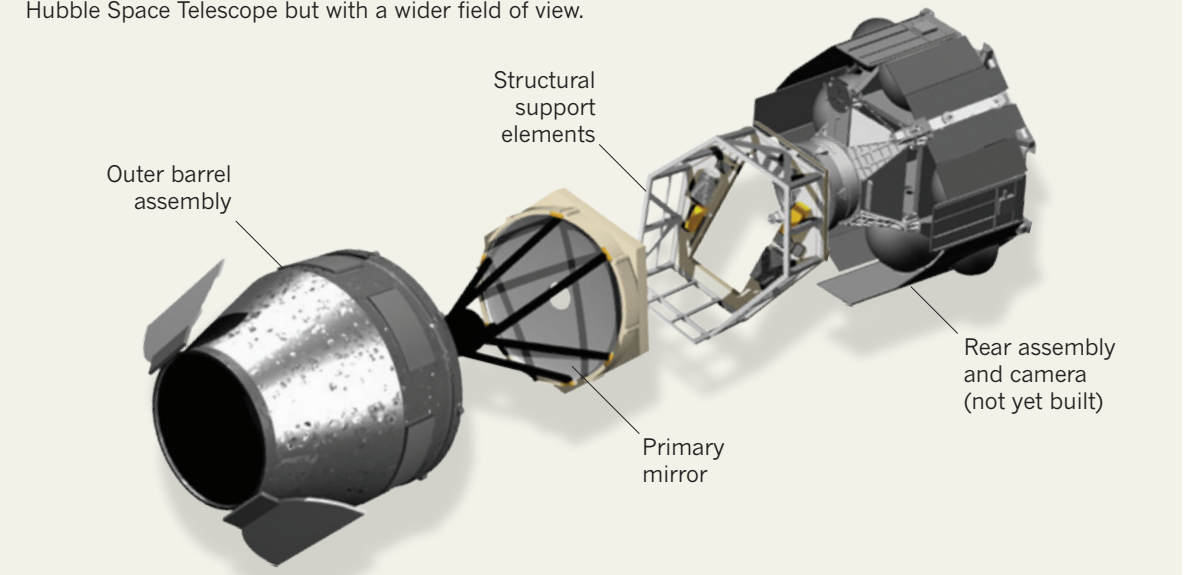

\section{FIELD OF VIEW}

Camera detectors built for the NRO telescope could potentially survey much bigger swathes of the sky than Hubble's best cameras.
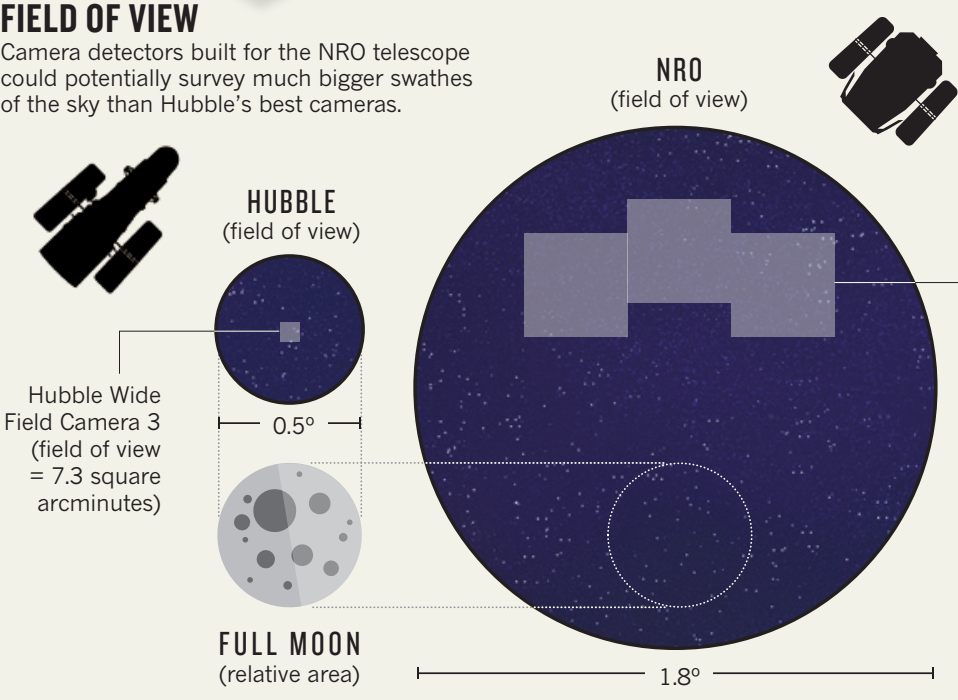

Proposed NRO camera (field of view $=1,350$ square arcminutes)

Infrared Space Telescope (WFIRST), the topranked mission in the 2010 astronomy decadal survey. Before the NRO telescopes came along, astronomers were aiming for a wide-field 1.3metre survey telescope to search for the imprint of dark energy, to find exoplanets and to study star-forming regions of the Galaxy. WFIRST was not expected to fly until the mid 2020s. But with a telescope already in hand, the NRO version of a WFIRST mission could conceivably launch at the end of the decade - potentially challenging Europe's Euclid space telescope, a dark-energy mission scheduled to fly in 2019.

The NRO telescope suits the WFIRST mission because it has a much wider field of view than Hubble (see 'Anatomy of a gift'), which makes it perfect for spotting the thousands of supernovae and millions of galaxies needed to pin down dark energy. And at a workshop held last month at Princeton University in New Jersey, Gary Matthews, the director of astronomy at ITT Exelis, released data showing how smoothly the telescope's mirror had been polished. "The mirror is about 
as good as Hubble's," he says.

The telescope's supporting structure is made of a resin that resists distortions caused by temperature changes, which will help keep the main mirror stable. Active control of a secondary mirror could adjust it to correct for any distortions due to the main mirror, further sharpening the telescope's optics.

"This telescope was clearly designed to produce very stable images," says Matt Mountain, director of the Space Telescope Science Institute in Baltimore, Maryland. And extremely stable images will be crucial for the success of one particular dark-energy technique - weak lensing - which looks for subtle distortions in the shapes of galaxies due to intervening dark matter (see Nature 489, 190-191; 2012).

Astronomers acknowledge that the telescope will not be ideal for studying the most distant galaxies in the Universe, which are visible only in the infrared. That requires a system for cooling the mirror but, unlike the proposed WFIRST instrument, the NRO telescope is designed to work at room temperature. However, its larger mirror will have so much more light-gathering power that it will be able to spot many more faint objects nearer to hand, even if it can't stare as deeply into space.

\section{COSTS AND BENEFITS}

Some astronomers, however, are questioning whether the value of the free hardware - each NRO telescope is worth at least US\$250 million - can compensate for the extra costs entailed in going from a 1.3-metre mission to a 2.4-metre mission, which will require a larger rocket and a larger camera. Although the WFIRST mission was expected to cost $\$ 1.5$ billion, one NASA estimate puts the NRO option at $\$ 1.75$ billion.

But Princeton astronomer David Spergel, who organized the workshop, believes that that figure underestimates the savings to be made by using the NRO scope. Not having to cast and polish a primary mirror avoids a long, labour-intensive process requiring an army of technicians, he says. Spergel thinks that a $\$ 1.6$-billion mission is realistic.

He would bump up the cost another \$200 million, however, to add an instrument that could take advantage of the extra lightgathering capability of the NRO telescope. This would be a coronagraph, which can block the light of a star while still revealing the dim glow of orbiting planets. WFIRST was going to search for exoplanets by detecting gravitational-lensing events: distortions in the light of a background star caused by a large planet orbiting another star in the foreground. But that method would not easily allow astronomers to detect planets that orbit close enough to be within a star's habitable zone. Astronomers would also prefer to collect a planet's reflected light directly. Mountain says that a modern coronagraph on NRO-WFIRST might be able to detect a Neptune-sized planet. It could also survey the dust that shrouds many stars and that could block small planets from view. Knowing how ubiquitous and severe the dust problem is would help exoplanet astronomers work out just how big their next big ask - a mission to directly detect Earth-like planets has to be.

\section{HUMAN INVOLVEMENT}

One way to reduce the cost of the NROWFIRST mission for NASA's astrophysics division would be to launch it on one of the new fleet of rockets that NASA will be eager to test at the end of the decade as it moves beyond the now-grounded space shuttles. But that would involve NASA's human space programme, an option that the science-definition team has been asked to consider. It could mean moving the mission from its intended orbit around the Sun - at a dynamically stable spot known as a Lagrangian point some 1.5 million kilometres beyond Earth's orbit - to a geostationary orbit about 36,000 kilometres above Earth (still much further out than Hubble). The geostationary option would be within reach of a wider variety of rockets - and of potential servicing missions by astronauts.

Although a Lagrangian point is a better spot for astrophysical observations because, for example, it lies outside Earth's radiation belts and has a more constant temperature environment, a geostationary orbit would allow much higher data-transfer rates, which would be particularly important in a survey mission accumulating vast amounts of data.

Astrophysicists believe that they are following the most sensible path for repurposing the NRO hardware, but they are aware that $\begin{array}{ll}\text { "It seemed too } & \begin{array}{l}\text { other space scien- } \\ \text { tists wouldn't mind }\end{array} \\ \text { good to be true. } & \text { a crack at it too. One } \\ \text { Things like } & \text { idea presented at } \\ \text { this just don't } & \text { the Princeton work- } \\ \text { drop on your } & \text { shop was to investi- } \\ \text { doorstep." } & \text { gate Earth's aurora } \\ & \text { and ionosphere by }\end{array}$ observing the edge of Earth from a vantage point within the orbit of the Moon. This proposal might need special approval, however, as one of the NRO's stipulations for donating the telescopes was that they would not be used to look at Earth. Such a large telescope could also be useful in planetary science, to search for asteroids likely to pass close to Earth or to study the faint objects beyond Neptune's orbit.

But there is a second telescope that could support these ideas, and there are assorted loose parts, including a primary mirror, for a third telescope, testimony to the NRO's lavish funding. Touring the ITT Exelis facility, Dressler was struck by the number of huge chambers designed to test space telescopes under vacuum. This was a place ready to stamp out Hubble-sized telescopes by the dozen, he says. "It makes you a little jealous," he says. "It's kind of neat and kind of sad." 